\title{
The free radical scavengers edaravone and tempol suppress experimental dextran sulfate sodium-induced colitis in mice
}

\author{
YOSHIO ARAKI $^{1}$, HIROYUKI SUGIHARA $^{2}$ and TAKANORI HATTORI ${ }^{2}$ \\ ${ }^{1}$ Department of Internal Medicine, Biwako Youikuin Hospital, 7-7-2 Ohgaya, Otsu 520-2144; \\ ${ }^{2}$ First Department of Pathology, Shiga University of Medical Science, Seta Tsukinowa, Otsu 520-2192, Shiga, Japan
}

Received September 16, 2005; Accepted November 18, 2005

\begin{abstract}
Recent studies have suggested that the enhanced release of reactive oxygen species (ROS) plays an important role in the pathogenesis of clinical inflammatory bowel disease (IBD), such as ulcerative colitis and Crohn's disease. In the present study, we investigated the effects of the free radical scavengers edaravone and tempol in the development of experimental dextran sulfate sodium (DSS)-induced colitis in mice. Male BALB/cA mice were fed $4 \%$ (w/w of diet) DSS in standard powder chow for 8 days. Edaravone, tempol, or vehicle saline were then injected subcutaneously twice per day. After the experimental period, the colonic length, histological damage score, and mucosal myeloperoxidase (MPO) and serum interleukin-6 (IL-6) levels were measured. Edaravone $(15 \mathrm{mg} / \mathrm{kg} /$ day $)$ and tempol $(5-15 \mathrm{mg} / \mathrm{kg} /$ day $)$ suppressed the colonic shortening and the damage score. In particular, tempol at $15 \mathrm{mg} / \mathrm{kg} /$ day significantly attenuated the colonic shortening and damage score. Edaravone and tempol suppressed the serum IL-6 levels, and significantly suppressed the increased colonic MPO levels. These results strongly support the involvement of ROS in the pathogenesis of DSS-induced colitis. A clinical effect for edaravone and tempol in IBD patients is strongly expected.
\end{abstract}

\section{Introduction}

Human inflammatory bowel disease (IBD), including Crohn's disease (CD) and ulcerative colitis (UC), is a chronic, relapsing, and remitting condition that exhibits various features of immunological inflammation. IBD is characterized by an infiltration of large numbers of neutrophils, monocytes, and lymphocytes into the large and/or small intestine, and also by diarrhea, bloody stools, abdominal pain, weight loss, anemia, and leukocytosis. IBD affects at least 1 in 1000 people in Western countries (1). Despite numerous studies on this

Correspondence to: Dr Yoshio Araki, 6-13-20 Aoyama, Otsu, Shiga 520-2101, Japan

E-mail: bileacidaraki@hotmail.com

Key words: dextran sulfate sodium-induced colitis, reactive oxygen species, free radical scavenger disease, the etiology and pathogenic mechanisms responsible for IBD remain poorly understood. At present, corticosteroids and sulfasalazine are commonly used for the treatment of IBD patients (2). However, the use of these agents is limited by their adverse effects (3).

Experimental animal models of IBD have been developed in order to investigate the mechanisms responsible for IBD, and to improve the medical therapy for this disease. In the most commonly used model, colitis is induced by administering polysaccharides such as dextran sulfate sodium (DSS) or carrageenan. DSS is a heparin-like polysaccharide containing approximately $17 \%$ sulfur, with up to three sulfate groups per glucose molecule (4). It has been reported that DSS inducedcolitis exhibits some of the clinical and histological features of UC. The precise mechanisms responsible for DSS-induced colitis, however, also remain unclear.

On the other hand, recent studies have suggested that the enhanced release of reactive oxygen species (ROS) plays an important role in the pathogenesis of DSS-induced colitis $(5,6)$. Therefore, the therapeutic effects of antioxidants on colitis such as the effect of melatonin on dinitrobenzene sulfonic acid-induced colitis (7) or DSS-induced colitis (8) have been investigated, as well as the effect of vitamin $E$ on trinitrobenzenesulfonic acid (TNBS)-induced experimental colitis (9) or DSS-induced colitis (10), and the effect of a superoxide dismutase-mimeric on TNBS colitis (11) or DSSinduced colitis (12). In our previous study, we also reported the beneficial effects of the free radical scavenger edaravone on DSS-induced rat colitis (13). Edaravone was utilized for the first time clinically during the acute phase of stroke as a free radical scavenger. On the other hand, the membranepermeable radical scavenger tempol was also reported to attenuate dinitrobenzene sulfonic acid-induced colitis (14). Tempol (4-hydroxy-2, 2, 6, 6-tetramethylpiperidine-N-oxyl) is a stable piperidine nitroxide, and functions as a stable free radical with low molecular weight, which permeates biological membranes and scavenges superoxide anions in vitro (15).

The purpose of the present study was: a) to investigate whether edaravone exerts the same therapeutic effects on the DSS-induced mouse colitis model as in the DSS rat colitis model; b) to investigate whether tempol exerts the same therapeutic effects on DSS-induced mouse colitis as dinitrobenzene sulfonic acid-induced colitis; and c) to compare the therapeutic effects between edaravone and tempol. 


\section{Materials and methods}

Animals. Specific pathogen-free male BALB/cA mice at 6 weeks old were purchased from Nippon Clea Inc. (Tokyo, Japan). They were housed in a room with controlled temperature $\left(20-22^{\circ} \mathrm{C}\right)$, humidity $(50-60 \%)$ and a preset light-dark cycle $(12: 12 \mathrm{~h})$. The experimental protocol was approved by the Animal Care and Use Committee of the Shiga University of Medical Science.

Free radical scavengers. Edaravone was kindly donated by Pharma Corporation (Tokyo, Japan). Edaravone was originally produced as a new anti-stroke agent in Japan, and has high antioxidant and anti-ischemic activities $(16,17)$. Tempol (4-hydroxy-2,2,6,6-tetramethyl-piperidinoxyl-N-oxyl) was purchased from Sigma-Aldrich (St. Louis, MO). Edaravone was dissolved in $1 \mathrm{~N} \mathrm{NaOH}$ and titrated to $\mathrm{pH} 7.2$ with $1 \mathrm{~N}$ $\mathrm{HCl}$. Tempol was dissolved in sterile water.

DSS-induced colitis, and edaravone or tempol treatment. DSS-induced colitis was provoked by the method previously described $(18,19)$. The mice were allowed food and drinking water ad libitum. After 7 days of acclimatization, the mice were fed the standard diets (MF, Oriental Yeast Co., Ltd., Tokyo, Japan) containing 4\% (w/w of diet) DSS (molecular weight 5000, total sulfur 15.0-20.0\%; Wako Pure Chemical, Osaka, Japan) for another 8 days. During the experimental period, food intake was measured daily. Concomitantly, $0.1 \mathrm{ml}$ of edaravone $(15 \mathrm{mg} / \mathrm{kg} /$ day $)$ or tempol $(5 \mathrm{mg} / \mathrm{kg} /$ day or $15 \mathrm{mg} / \mathrm{kg} /$ day) were injected subcutaneously twice per day (7 a.m. and 7 p.m.). These doses were based on those used in previously reported studies $(13,14)$. In the vehicletreated group, $0.2 \mathrm{ml}$ of saline was injected subcutaneously twice per day $(n=5)$.

Colonic length and damage score. On the final day of the experiment, the mice were anesthetized with an intraperitoneal injection of pentobarbital sodium $(30 \mathrm{mg} / \mathrm{kg})$. After blood samples were collected by cardiac puncture, the mice were sacrificed by cervical dislocation. The entire colon was removed, and the length was measured. A tissue specimen was removed $0.5 \mathrm{~cm}$ from the anal margin, fixed in Carnoy's fixative and embedded in paraffin. Histological samples were then cut into $5 \mu \mathrm{m}$ thick sections, and stained with hematoxylin and eosin (HE) after de-paraffinization. The mucosal damage was evaluated independently by two investigators blinded to the study groups, and determined according to a previously described method (20). These scores derived from the two investigators were then averaged for a composite score. The following three parameters were used: surface epithelial loss, crypt destruction and inflammatory cell infiltration into the mucosa. A score of 0-4 was assigned to each of the three parameters according to the extent and severity of the change as follows: 0 , no change; 1 , localized and mild; 2, localized and moderate; 3 , extensive and moderate; and 4, extensive and severe. The sum of the scores from these three parameters was defined as the mucosal damage score for each animal.

Mucosal myeloperoxidase (MPO). The levels of intestinal myeloperoxidase (MPO), a biochemical marker of MPO- positive granulocytes, were measured using a commercially available ELISA kit. Briefly, a mucosal scraping from the remnant colon was placed in $1 \mathrm{ml}$ of a hexadecyltrimethylammonium bromide solution $(0.5 \%, \mathrm{w} / \mathrm{w})$. The solution was then homogenized and sonicated, and the resulting homogenate was subjected to three rapid cycles of freezing and thawing. The samples were centrifuged in a microfuge to remove any insoluble material (14000 rpm for $20 \mathrm{~min}$ ), and the supernatants were used for the measurement of MPO levels using a commercially-available Myeloperoxidase ELISA kit (Calbiochem, CA). The protein content of the supernatant was also measured using a Bio-Rad Protein Assay (Bio-Rad Laboratories Ltd., CA).

Serum interleukin-6 (IL-6) levels. In DSS-induced colitis, the development of colitis is strongly associated with IL-6 production (21). Therefore, we measured the serum IL-6 levels in mice using a commercially-available ELISA kit mouse-IL-6 (Biosource, CA).

Statistics. The results were presented as means \pm SEM. The variance was analyzed by the Bartlett test. Subsequently, a one-factor ANOVA and Fisher's PLSD test was performed to compare the means of the normally distributed data. The Kruskal-Wallis test and Dunn's procedure as a multiple comparison procedure were also performed to compare the means of the non-parametric or abnormally distributed data. Differences were regarded as statistically significant when the $\mathrm{p}$-values were $<0.05$.

\section{Results}

Colonic length and damage score. None of the rats in any of the groups died during the experimental period. There were no significant differences in food intake between the edaravone-treated, tempol-treated, and vehicle-treated mice. DSS administration decreased significantly the colonic length as compared to the control mice (Fig. 1A). Edaravone and tempol attenuated the colonic shortening due to DSS administration. In particular, tempol significantly attenuated the colonic shortening. On HE staining, there was obvious evidence of inflammatory cell infiltration into the mucosa and submucosa in the distal portions of all groups. Entire crypt loss, surface epithelial loss and mucosal edema were also evident. The total damage score as quantified by the scoring system is shown in Fig. 1B. DSS administration significantly increased the damage score as compared to the control mice. Edaravone and tempol both reduced the damage score due to DSS administration. In particular, tempol significantly reduced the damage score at a dose of $15 \mathrm{mg} / \mathrm{kg} /$ day.

Mucosal MPO activity and serum IL-6 levels. The mucosal MPO activities in the control, vehicle-treated, edaravonetreated, and tempol-treated mice are shown in Fig. 2A. DSS administration significantly increased the mucosal MPO activities as compared to the control mice. Both edaravone and tempol significantly attenuated the increased colonic MPO activity induced by the DSS administration. With respect to the serum IL-6 levels, DSS administration significantly 


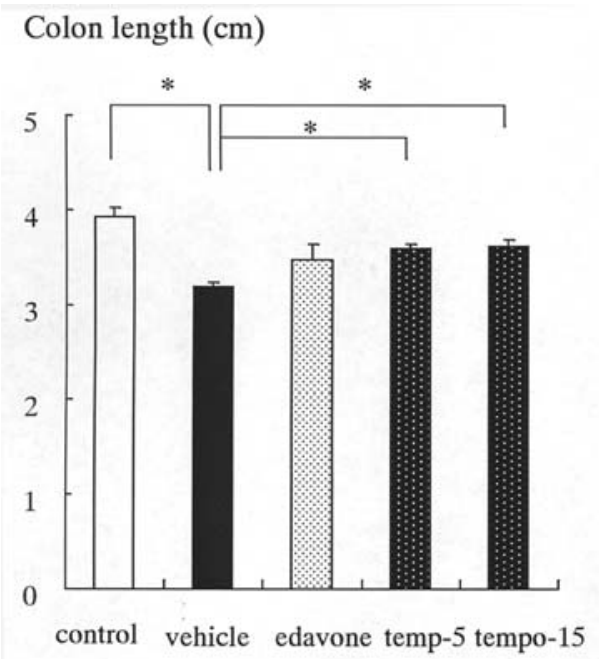

(A)
Damage score

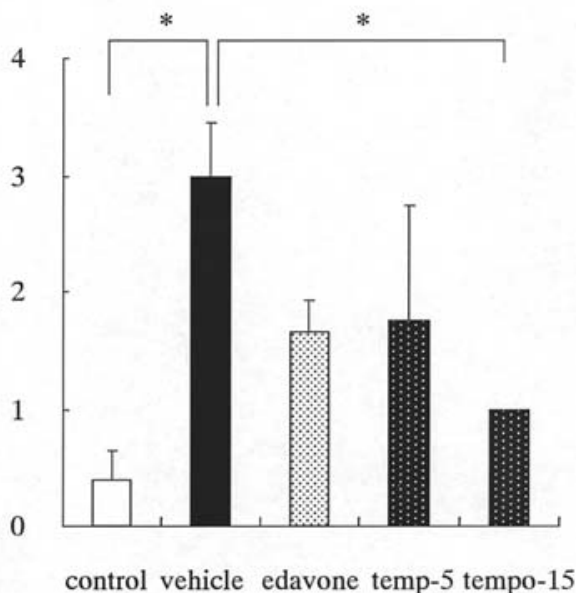

(B)

Figure 1. Colonic length and mucosal damage score in each group. After the mice were fed the experimental diets, $0.1 \mathrm{ml}$ of edaravone (15 mg/kg/day) or tempol $(5 \mathrm{mg} / \mathrm{kg} /$ day or $15 \mathrm{mg} / \mathrm{kg} /$ day $)$ was injected subcutaneously twice per day for 8 days. The colinic length was measured and the mucosal damage score was quantified by the scoring system described in the text. (A) Colonic length and (B) mucosal damage score in the control, vehicle-treated, edaravonetreated, and tempol-treated mice. The values are expressed as means \pm SEM. ${ }^{*} \mathrm{p}<0.05$.

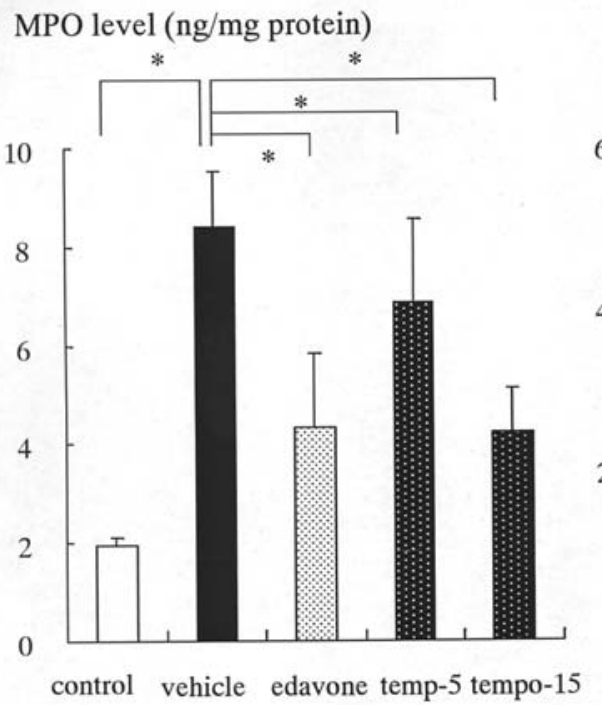

(A)

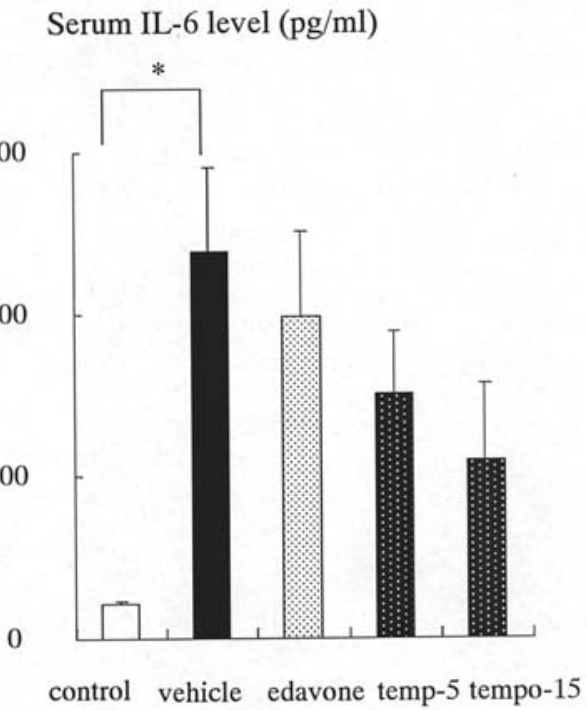

(B)

Figure 2. Mucosal MPO and serum IL-6 levels. A mucosal scraping from the colon was placed in hexadecyltrimethylammonium bromide solution, homogenized, and sonicated. Then, the resulting homogenate was subjected to rapid cycles of freezing and thawing, centrifuged. The supernatants were used for the measurement of MPO levels using a commercially-available ELISA kit. The protein content of the supernatant and serum IL-6 levels were also measured using a commercially available ELISA kits. The values are expressed as means \pm SEM. ${ }^{*} \mathrm{p}<0.05$.

increased the serum IL-6 levels as compared to the control mice. Again, both edaravone and tempol suppressed the serum IL-6 levels, but these differences did not reach statistical significance (Fig. 2B).

\section{Discussion}

The precise mechanisms responsible for DSS-induced colitis are still unclear. However, we have reported the therapeutic effects of dietary fiber on this colitis model and clinical IBD
(22-25). These results strongly suggest that environmental factors in the gut lumen play very important roles in maintaining healthy gut function. In addition, recent studies have suggested that ROS and nitric oxide may play a role in the pathogenesis of DSS-induced colitis $(5,6)$. In this context, previous studies have reported that radical scavengers exerted therapeutic effects against DSS-induced, TNBS-induced, and other kinds of colitis (7-15). In this colitis model, the candidate for the production of the ROS has been suggested to be the infiltrating neutrophils (17). 
In the present study, edaravone could not completely prevent the development of DSS-induced colitis in mice. However, edaravone ameliorated the inflammation in this model, as we previously reported in a rat model (13). Tempol did not either completely prevent the development of DSSinduced colitis in the mice. However, tempol ameliorated the inflammation, as it did against dinitrobenzene sulfonic acidinduced colitis (14). Taken together, one of the pathogenic mechanisms responsible for DSS-induced colitis may be ROS formation, and these results support previous studies in which ROS are suggested to play a role in the pathogenesis of DSS-induced colitis $(5,6)$.

Edaravone is the only drug used clinically as a free radical scavenger, and has been used widely in stroke patients at a dose of $1 \mathrm{mg} / \mathrm{kg} / \mathrm{day}$. In our previous study, we recognized that edaravone at a clinically-relevant $(1 \mathrm{mg} / \mathrm{kg} /$ day $)$ dose exerted a detectable therapeutic effect, but edaravone at a dose of $20 \mathrm{mg} / \mathrm{kg} /$ day exerted the strongest therapeutic effects on DSS-induced colitis without any organic abnormalities or side effects (13). Tempol is also a free radical scavenger, but no previous clinical studies have been reported on it. In the present study, therefore, we set the dose of edaravone and tempol at $15 \mathrm{mg} / \mathrm{kg} /$ day according to a previous study using an animal model of dinitrobenzene sulfonic acid-induced colitis (14). Tempol exerted its therapeutic effects at a minimal dose of $5 \mathrm{mg} / \mathrm{kg} /$ day. In addition, it seems that there is a dose-response relationship between the amount of tempol used and how effectively the drug exerts its therapeutic benefits. Effective therapies have long been needed for clinical UC, and edaravone and tempol are expected to exert similar therapeutic effects on clinical UC patients.

In conclusion, the free radical scavengers edaravone and tempol attenuated DSS-induced colitis. These observations strongly support the involvement of ROS in the pathogenesis of DSS-induced colitis. Beneficial clinical effects in the treatment of UC patients are strongly anticipated as a consequence of this novel strategy.

\section{References}

1. Fiocchi C: Inflammatory bowel disease: etiology and pathogenesis. Gastroenterology 115: 182-205, 1998.

2. Stephen BH, Samuel M and David BS: The pharmacology of anti-inflammatory drugs in inflammatory bowel disease. In: Inflammatory Bowel Disease. 4th edition. Joseph BK and Roy GS (eds). Williams and Wilkins, Baltimore, pp664-694, 1995.

3. Stephen BH, Samuel M and David BS: The pharmacology of anti-inflammatory drugs in inflammatory bowel disease. In: Inflammatory Bowel Disease. 4th edition. Joseph BK and Roy GS (eds). Williams and Wilkins, Baltimore, pp643-663, 1995.

4. Ricketts CR: Dextran sulphate-a synthetic analogue of heparin. Biochem J 51: 129-133, 1952.

5. Blackburn AC, Doe WF and Buffinton GD: Salicylate hydroxylation as an indicator of hydroxyl radical generation in dextran sulfate-induced colitis. Free Radic Biol Med 25: 305-313, 1998 .

6. Dykens JA and Baginski TJ: Urinary 8-hydroxydeoxyguanosine excretion as a non-invasive marker of neutrophil activation in animal models of inflammatory bowel disease. Scand J Gastroenterol 3: 628-636, 1998.

7. Cuzzocrea S, Mazzon E, Serraino I, Lepore V, Terranova ML, Ciccolo A and Caputi AP: Melatonin reduces dinitrobenzene sulfonic acid-induced colitis. J Pineal Res 30: 1-12, 2001.
8. Pentney PT and Bubenik GA: Melatonin reduces the severity of dextran-induced colitis in mice. J Pineal Res 19: 31-39, 1995.

9. Ademoglu E, Erbil Y, Tam B, Barbaros U, Ilhan E, Olgac V and Mutlu-Turkoglu U: Do vitamin E and selenium have beneficial effects on trinitrobenzenesulfonic acid-induced experimental colitis? Dig Dis Sci 49: 102-108, 2004.

10. Carrier J, Aghdassi E, Cullen J and Allard JP: Iron supplementation increases disease activity and vitamin E ameliorates the effect in rats with dextran sulfate sodium-induced colitis. J Nutr 132: 3146-3150, 2002.

11. Cuzzocrea S, Mazzon E, Dugo L, Caputi AP, Riley DP and Salvemini D: Protective effects of M40403, a superoxide dismutase mimetic, in a rodent model of colitis. Eur J Pharmacol 432: 79-89, 2001.

12. Ogawa Y, Kanatsu K, Iino T, Kato S, Jeong YI, Shibata N, Takada K and Takeuchi K: Protection against dextran sulfate sodium-induced colitis by microspheres of ellagic acid in rats. Life Sci 71: 827-839, 2002.

13. Araki Y, Andoh A and Fujiyama Y: The free radical scavenger edaravone suppresses experimental dextran sulfate sodiuminduced colitis in rats. Int J Mol Med 12: 125-129, 2003.

14. Cuzzocrea S, McDonald MC, Mazzon E, Dugo L, Lepore V, Fonti MT, Ciccolo A, Terranova ML, Caputi AP and Thiemermann C: Tempol, a membrane-permeable radical scavenger, reduces dinitrobenzene sulfonic acid-induced colitis. Eur J Pharmacol 406: 127-137, 2000.

15. Laight DW, Berthelon JJ, Andrews TJ, Carrier MJ and Anggord EE: Assessment of superoxide anion scavenging activity of some common spin traps at physiological temperature and $\mathrm{pH}$ by microassay. Br J Pharmacol 122: 130-135, 1997.

16. Watanabe T, Yuki S, Egawa M and Nishi H: Protective effects of MCI-186 on cerebral ischemia: possible involvement of free radical scavenging and antioxidant actions. J Pharmacol Exp Ther 268: 1597-1604, 1994.

17. Watanabe T and Egawa M: Effects of antistroke agent MCI-186 on cerebral arachidonate cascade. J Pharmacol Exp Ther 271: 1624-1629, 1994.

18. Araki Y, Andoh A, Takizawa J, Takizawa W and Fujiyama Y: Clostridium butyricum, a probiotic derivative, suppresses dextran sulfate sodium-induced experimental colitis in rats. Int J Mol Med 13: 577-580, 2004.

19. Kanauchi O, Serizawa I, Matsumura T, Fukuda Y and Satomi M: Evaluation of antigenicity of germinated barley foodstuff for the treatment of ulcerative colitis in a chronic murine colitis model. Int J Mol Med 7: 143-147, 2001.

20. Oda T: Role of mast cells in dextran sulfate sodium-induced experimental colitis in rats. J Kyoto Pref Univ Med 104: 1069-1082, 1995.

21. Suzuki A, Hanada T, Mitsuyama K, Yoshida T, Kamizono S, Hoshino T, Kubo M, Yamashita A, Okabe M, Takeda K, Akira S, Matsumoto S, Toyonaga A, Sata M and Yoshimura A: CIS3/ SOCS3/SSI3 plays a negative regulatory role in STAT3 activation and intestinal inflammation. J Exp Med 193: 471-481, 2001.

22. Araki Y, Fujiyama Y, Andoh A, Koyama S, Kanauchi O and Bamba T: The dietary combination of germinated barley foodstuff plus Clostridium butyricum suppresses the dextran sulfate sodium-induced experimental colitis in rats. Scand J Gastroenterol 35: 1060-1067, 2000.

23. Hanai H, Kanauchi O, Mitsuyama K, Andoh A, Takeuchi K, Takayuki I, Araki Y, Fujiyama Y, Toyonaga A, Sata M, Kojima A, Fukuda M and Bamba T: Germinated barley foodstuff prolongs remission in patients with ulcerative colitis. Int J Mol Med 13: 643-647, 2004.

24. Kanauchi O, Mitsuyama K, Homma T, Takahama K, Fujiyama Y, Andoh A, Araki Y, Suga T, Hibi T, Naganuma M, Asakura H, Nakano H, Shimoyama T, Hida N, Haruma K, Koga H, Sata M, Tomiyasu N, Toyonaga A, Fukuda M, Kojima A and Bamba T: Treatment of ulcerative colitis patients by long-term administration of germinated barley foodstuff: Multi-center open trial. Int J Mol Med 12: 701-704, 2003.

25. Fukuda M, Kanauchi O, Araki Y, Andoh A, Mitsuyama K, Takagi K, Toyonaga A, Sata M, Fujiyama Y, Fukuoka M, Matsumoto Y and Bamba T: Prebiotic treatment of experimental colitis with germinated barley foodstuff: A comparison with probiotic or antibiotic treatment. Int J Mol Med 9: 65-70, 2002 . 